\title{
Performance Evaluation of Face Recognition system by Concatenation of Spatial and Transformation Domain Features
}

\author{
Raveendra K \\ Research Scholar, Department of ECE, Global Academy of Technology, Bangalore-560098 \\ Assistant Professor, Government Engineering College, K R Pet- 571426, Karnataka, India \\ E-mail: raveendrakit@gmail.com
}

\section{Ravi J}

Professor, Department of ECE

Global Academy of Technology, Bangalore-560098, Karnataka, India

E-mail: gnkereravi@gmail.com

Received: 14 September 2020; Accepted: 30 October 2020; Published: 08 February 2021

\begin{abstract}
Face biometric system is one of the successful applications of image processing. Person recognition using face is the challenging task since it involves identifying the $3 \mathrm{D}$ object from $2 \mathrm{D}$ object. The feature extraction plays a very important role in face recognition. Extraction of features both in spatial as well as frequency domain has more advantages than the features obtained from single domain alone. The proposed work achieves spatial domain feature extraction using Asymmetric Region Local Binary Pattern (ARLBP) and frequency domain feature extraction using Fast Discrete Curvelet Transform (FDCT). The obtained features are fused by concatenation and compared with trained set of features using different distance metrics and Support Vector Machine (SVM) classifier. The experiment is conducted for different face databases. It is shown that the proposed work yields $95.48 \%$ accuracy for FERET, $92.18 \%$ for L-space $\mathrm{k}, 76.55 \%$ for JAFFE and $81.44 \%$ for NIR database using SVM classifier. The results show that the proposed system provides better recognition rate for SVM classifier when compare to the other distance matrices. Further, the work is also compared with existing work for performance evaluation.
\end{abstract}

Index Terms: FDCT, ARLBP, Support Vector Machine, Euclidean Distance, Recognition.

\section{Introduction}

With the advancement in the internet technology, privacy concern, networking, communication and mobile systems, there is a need for reliable and secure authentication techniques. Increasing demand and deployment of biometric systems are identified in various domains like banking, forensic applications and airports etc, has begun to conquer more attention as an important system for ascertaining an individual's identity in various places.

The term Biometric is mainly originated from Greek word Bio means life and metrikos means measure [1]. It authenticates the person depending on his/her unique features. Biometric traits are classified based on behavioral and/or physiological traits. Behavioral characteristics are related to the behavior of a person includes signature, keystroke and gait recognition system. Whereas physiological characteristics are related to shape of the body which includes face, finger, iris, palm, hand vein and DNA. The latest term in this field is soft biometric, which reveals certain data about the user, is combined with his/her own biometric identity to provide a stronger authentication method. Earlier method of authentication was deployed using two approaches. In the first each user has his/her own User ID, smart cards or badges which possess very less security as these entities can be easily stolen, replicated or shared. Second approach is to recognize a person based on his/her PIN or a password which is assigned for him/her. This approach is also not secure since the PIN or password can be forgotten and easily hacked by a third party for unauthorized access. Biometric based security systems can easily solve the problems of earlier methods since the persons are recognized who they are not by remembering or carrying any identity gadgets with them. Biometric traits are very difficult to forge, imitate, share, forget or guess etc. Nowadays though there are various biometric systems are available but not all biometric systems does not fit for all types of applications. The type of biometric is selected based on the application requirement and the recognition accuracy.

Face biometric system is one of the successful applications of image processing. Person recognition using face is the challenging task since it involves identifying the $3 \mathrm{D}$ object from $2 \mathrm{D}$ object. Basically Face recognition system 
mainly includes four steps namely (i) Facial image capturing and storage: The facial images from each database contains face images of multiple people with different pose and expression variations of the faces. (ii) Image Preprocessing: Face Detection is performed to eliminate the background noise and extracting the region of interest (i,e, Face).The detected face is cropped, resized and converted to gray color for further processing. (iii) Feature Extraction: The image features are extracted in both spatial and transform domain. The spatial domain includes extracting statistical features, edge features, local pixel relativity etc. The transform domain includes Discrete wavelet Transform Features (DWT), Fast Fourier Transform Features (FFT), Curvelet transform Features, Contourlet transform features, Shearlet transform features etc. (iv) Classification: The classification involves matching a test features with trained set of features using different classifiers like Euclidean distance (ED), Support Vector Machine (SVM), City Block, Chisquare, Cosine Correlation, Spearman, Artificial neural Network (ANN) etc.

Many traditional algorithms deal with extracting features either in spatial or transformation domain. Use of any single domain has advantages in terms of speed of execution but in the other hand it is proven that the use of hybrid techniques (both spatial and transformation domain) greatly increases the recognition rate. With the hybrid methods one can enhance the recognition performance by preserving the strengths. The proposed paper achieves spatial domain feature extraction using ARLBP and frequency domain feature extraction using FDCT. The obtained features are fused by concatenation for classification. Section 2 reveals the study on previous work on face recognition which includes different single and hybrid techniques. Section 3 describes the proposed methodology of recognition system and its working process. Section 4 gives dataset preparation, Section 5 describes the preprocessing task, Section 6 describes the feature extractions, Section 7 is about classification methods used for matching ,tech the experimental results and its comparison with different distance metrics. 8 describes the experimental results and its comparison with different distance metrics. Conclusion are provides at the final section

\section{Literature Survey}

Face recognition algorithm at low resolution using Local Binary Patterns Histogram (LBPH) is implemented in Literature [2]. The algorithm was tested on LR500 dataset and found to operate better at the minimum low resolution of $35 \mathrm{px}$ during human motion. Face recognition algorithm implemented [3] to compare the performance of the system with good quality images, enhanced images and images added with Gaussian blur and motion blur. Harr features and Local Binary Patterns Histogram (LBPH) are used. It was found that the Gaussian blur is harder compared to motion blur for face recognition, also these blurs have negative impact on the performance of the system. Finally it's concluded that the enhanced images will be more likely recognized than blurred images. A multi-faces recognition technique with the Haar Cascade and Eigen Face method [4] to detect and recognize the straight faces has been introduced. This technique was tested to detect multiple faces in a single detection process. Dominant Rotated Local Binary Pattern (DRLBP) and Scale Invariant Feature Transform (SIFT) based facial recognition system is proposed in Literature [5].Here the face image is preprocessed before extracting the features by applying DRLBP and SIFT. The extracted features are compared using the Back Propagation Network (BPN).

Face recognition technique based on histogram equalization and convolution neural network introduced in [6]. In this technique face image is preprocessed using histogram equalization and the convolution neural network was built using deep learning frame work. The experiment was conducted on ORL database for recognition rate. In order to improve the face recognition rate [7] developed an algorithm of LBPH algorithm under illumination diversification, expression variation and attitude deflection. This algorithm considers the neighborhood of gray median of sample values rather than the intermediate values. The recognition rate of this algorithm is found to be superior to LBPH algorithm. A facial recognition algorithm using modified local ternary pattern (MLTP) with multiclass support vector classifier proposed in [8]. The literature classifies the MLTP features by an error correcting output code multiclass model with SVM for computing the face recognition rate. An edge based hybrid model for face detection using neural network classifier [9] and CANNY method is proposed. The classification of the faces and non-faces was done with the multilayer feed forward network. The facial features were extracted using CANNY method. The feasibility of the algorithm was tested by computing the recognition rate. An investigation on facial recognition was made with local directional and local binary patterns in [10]. From the results obtained by the trials, they concluded that the hybridization of local directional pattern (LDP) and extended Local binary pattern feature extraction with a voting classification algorithm improves the facial recognition accuracy. In [11] a model on human face gender identification system based on multi block local binary pattern (MBLBP) is proposed. The feature extracted from the proposed technique has overcome the short comings of traditional Local Binary Pattern (LBP) operators. The recognition capability of the proposed algorithm was found to better. In [12] proposed a model on deep neural network for face recognition. Feature selection, extraction and training was done using Convolutional Neural Networks (CovNets). The performance was measured on Yaleface database using softmax layer for classification. It was tested on single database with less number of images.

A model for the facial expression recognition by deploying the curvelet based LBP is designed in [13]. The curvelet transform was applied at specific scale and orientation on the face image. LBP was applied on the selected curvelets of bands to extract the dominant feature set. The recognition rate was computed with nearest neighbor 
classifier with the dissimilarity matrix Chi square. In [14] reported a model on face recognition and detection using random forest and combination of LBP and histograms of oriented gradients (HOG) features. Combination of the features improves the overall system accuracy with random forest classification. On the medium staff database he conducted four investigations.

A different classifier method is proposed to determine the face recognition rate in which feature extraction was done using PCA [15] and classification was done using Squared Euclidian Distance, Euclidian Distance, City-Block Distance by clustering the difference of training image with images set for each person and calculating the mean to check the face recognition rate. A deep learning based face recognition attendance system using deep convolutional neural network for face detection and recognition is developed in [16]. Higher accuracy was achieved with the small number of face images. In [17] have proposed a technique for faces recognition in which Pulse Coupled Neural Networks (PCNN) was employed to effectively suppress the noise and cluster the characteristic region of noisy faces, the PCA was used for dimensionality reduction and feature extraction. SVM is used for classification and recognition. An algorithm for face biometric with pose-invariant face databases is developed in [18]. The feature extraction from the image was carried out using the patch-wise self-similarity measure. The algorithm was tested on Far Infrared (FIR) database. Face recognition system based on speeded up robust features (SURF) and linear discriminant analysis (LDA) was introduced in [19]. DWT is applied on the dataset to produce a discrete output of nxn dimension matrix for input of size $\mathrm{n}$. LDA is applied on this matrix for dimensionality reduction and sub-space mapping. SURF is used for matching. The experiment was conducted on few number of images.

A facial image recognition algorithm in which the feature vectors of the encrypted face were extracted by applying Discrete Fourier Transform (DFT) on the original image to obtain complex co-efficient and then encrypted using Logistic chaotic map in [20]. Feature vectors of the encrypted face images in the database are compared with the acquired feature vectors and calculating the normalized cross correlation co-efficient (NC), the greatest value of NC gives recognized as matching.

A face recognition system based on facial landmarks presented in [21]. The recognition was analyzed by measuring slope and distance between facial landmarks. The analysis was done in statistical method as well as with classifiers. This technique can be successfully adopted for facial recognition from a low number of feature data and facial expression in different positions. Face recognition to recognize frontal and non-frontal faces has developed in [22]. Elliptical Local Binary Pattern (ELBP) was to extract the face features, these features are then decomposed by Discrete Wavelet Transform. Feature Classification was done using K-NN classifier. The model was tested on FERET database. The concept of face recognition using DT-CWT and LBP has been proposed in [23]. The coefficients of DTCWT have determined using 5 levels DT-CWT. Further, the obtained coefficients were organized into $3 \times 3$ matrixes. The final features of the face have been obtained using LBP on this 3 x 3 matrix. The test image features are compared with the image of the database through Euclidean Distance.

From the previous work of face recognition models $[2,3,4,5,6,7,12,13]$, it is observed that the spatial feature domain alone affects the performance of the system, since it produces spectral degradation because of not having fixed set basis vectors. Further, the traditional works are demonstrated using minimal database for performance evaluation.

In order to overcome this problem we have proposed a hybrid technique which combines Asymmetric Region Local Binary Pattern (ARLBP) technique to extract the set of features in spatial domain and Fast Discrete Curvelet Transform (FDCT) via wrapping to extract another set of face image features in transform domain. The experiment is also conducted individually for ARLBP and Curvelet alone. The results shows that the performance of the hybrid technique is superior when compare to the individual technique alone.

\section{Proposed Methodology}

The proposed work consists of both spatial and transforms domain techniques for extracting facial image features. The system incorporates Asymmetric Region Local Binary Pattern (ARLBP) technique to extract the set of features in spatial domain and Fast Discrete Curvelet Transform (FDCT) via wrapping to extract another set of face image features in transform domain. With the hybrid methods one can enhance the recognition performance by preserving the strengths. The ARLBP extracts the local structural feature information from the face image. This method has two advantages. First, the face can be represents as the set of feature vectors extracted from the local region, thereby reducing the dimensionality problem. Second, local features might be useful if some region of the face is occluded. The Curvelets via wrapping technique is used here because of simple, faster and less redundant than first generation curvelet transform. Further, use of curvelet has advantages than traditional wavelet transform as wavelet fails to extract the information near the curved, nonlinear like structures present in the face image there by reduces the system performance.

The extracted features from both the techniques are fused by concatenation to obtain final features. Different classifiers are used for matching. The block diagram of the proposed model is as shown in Fig.1. 


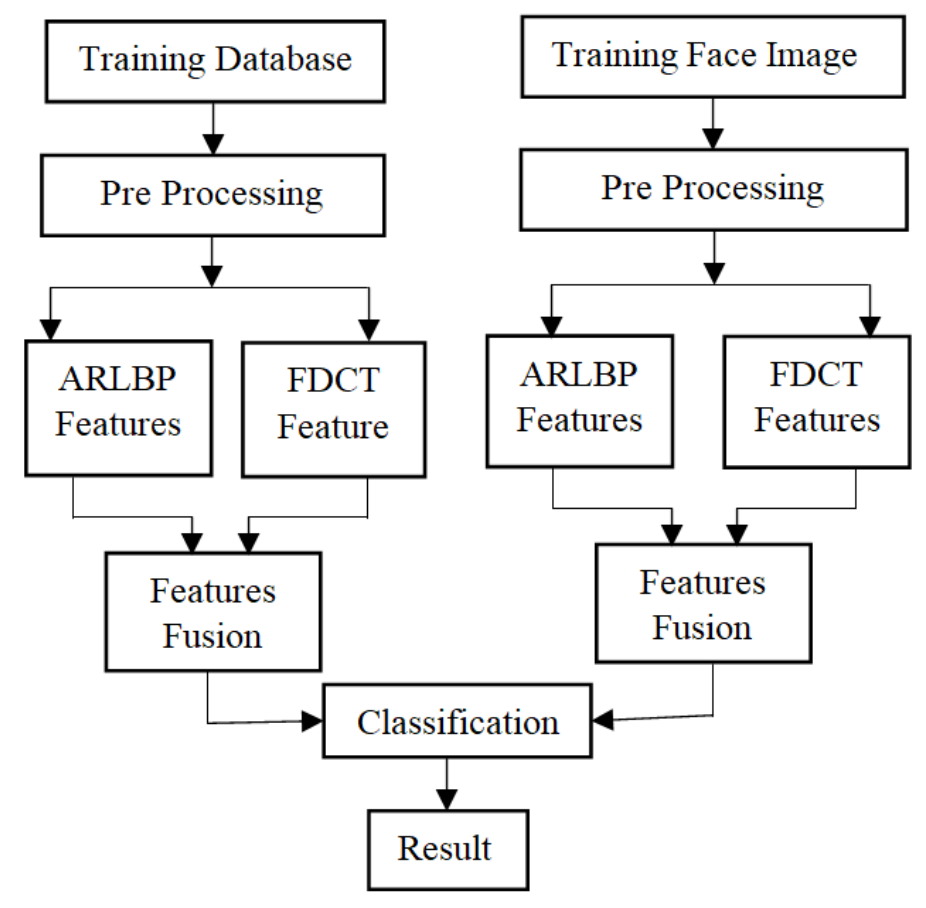

Fig.1. The block diagram of the proposed model

The proposed work includes two process. The first process is the Training Process, here we used face images from different databases such as L-Space K, Near Infrared Database (NIR) and FERET. The multi features are extracted for images present in the database and are stored. The Second process is the Recognition Process. In which randomly select the images from the database and also from out of the database in order evaluate the system performance.

\subsection{Training Process}

In the training process we used face images from different databases. After creating the database, the next step is face image preprocessing. Preprocessing involves extracting the region of interest (ROI) means "Face Detection" from the input face image. The detected face image is cropped for a fixed dimension and converted to grayscale image. ARLBP is applied for the cropped image in order to extract the spatial features. The ARLBP outputs feature vector of length $L$ for each face image. Further, for the original cropped face image Fast Discrete Curvelet Transform (FDCT) via wrapping is applied to extract another set of feature vector of length $N$ in transform domain. Finally, the face features from ARLBP and the features FDCT are concatenated to form final training feature of length $L+N$ vector and are stored. This process is performed for all face images present in the database to create feature space having a dimension $K \times(L+N)$, where $K$ is the total number of face images and $(L+N)$ is the length of each feature vector.

\subsection{Testing Process}

In the testing process, we randomly select the images from the database and also from out of the database for evaluation. As we discussed in the training process, testing process also involves face image preprocessing and multi feature extraction. Feature extraction involves extracting ARLBP and FDCT features for each face image and concatenating them to form testing feature vectors. Each testing face image features are compared with training features using distance metrics. The recognized face is one which has minimal difference error value. Different distance metrics are used for classification and the performance of each classifier is evaluated and tabulated.

\section{Dataset Preparation}

Each database has its own characteristics and the number of face images. The different database description and the number of images used for training and testing are given below

L-Spacek Database: The L-spacek database is considered for algorithm development because of its large variations in lightning, different orientations and expression. The database has 113 male persons containing 20 face images for each person. The system is trained for first 63 persons out 113 considering first 10 images per person for recognition. The recognition rate is calculated by testing the system with eleventh image of the 63 persons.

Near Infrared Database (NIR): The NIR database is considered for testing the algorithm because of its variations in intensity, illumination change and blurring effect, different pose and facial expressions. The database has total 115 
persons with 14 images for each person. The training data has been organized for first 20 persons from total 115 persons by considering first 6 images for each person. The recognition rate is calculated by testing the system with seventh image from same of 20 persons.

JAFFE Database: JAFFE Database is considered because of its identical/similar face images of all the persons. The database contains 213 images of 7 facial expressions posed by 10 Japanese female models. The system is trained for first 6 persons out of 10 persons and considering first 10 images of each person for recognition and to calculate the recognition rate.

FERET Database: FERET Database is used because of its larger size and contains face images having pose and light variations. From the database we have selected a total of 256 persons having 4 images per person with a total of 1024 images. We use the first image each individual as training set. Hence the total images in the training set is 256. The remaining three images from each individual we used for testing, so the size of test images is 768 .

\section{Preprocessing}

After creating the database, the next step is face image preprocessing. Preprocessing involves extracting the region of interest (ROI) means "Face Detection" from the input face image. The proposed work adopts Viola Jones algorithm [24] to detect the face from the complex background. This method uses Haar like features to extract features of both face and non-face regions. Haar features are small kernels having different shapes and scales which are used to detect the presence of the feature in the given image. Any redundancies of the obtained features were eliminated using Adaboost learning algorithm. Adaboost is machine learning algorithm which identifies best among all the features, but these features are called weak classifier, and to construct strong classifier by linearly combining weak classifier. Finally the cascade classifier contains strong classifier at different stages are used to detect the face in the given image. The Region of Interest (Face) is obtained by cropping and resized to 100x100 for all database.

\section{Feature Extraction}

\subsection{Asymmetric Region based Local binary Pattern (ARLBP)}

The basic Local Binary Pattern (LBP) operator of size 3x3 neighborhood has the limitation of capturing dominant features at the higher scale texture analysis, to overcome this limitation ARLBP operator is used.

The ARLBP is scalable and is capable of extracting dominant features at higher scales by considering higher values or rounded average intensities of the sub regions around the central pixel value. This will result in reducing the loss of texture information and increases the discriminative ability compared to LBP [25].

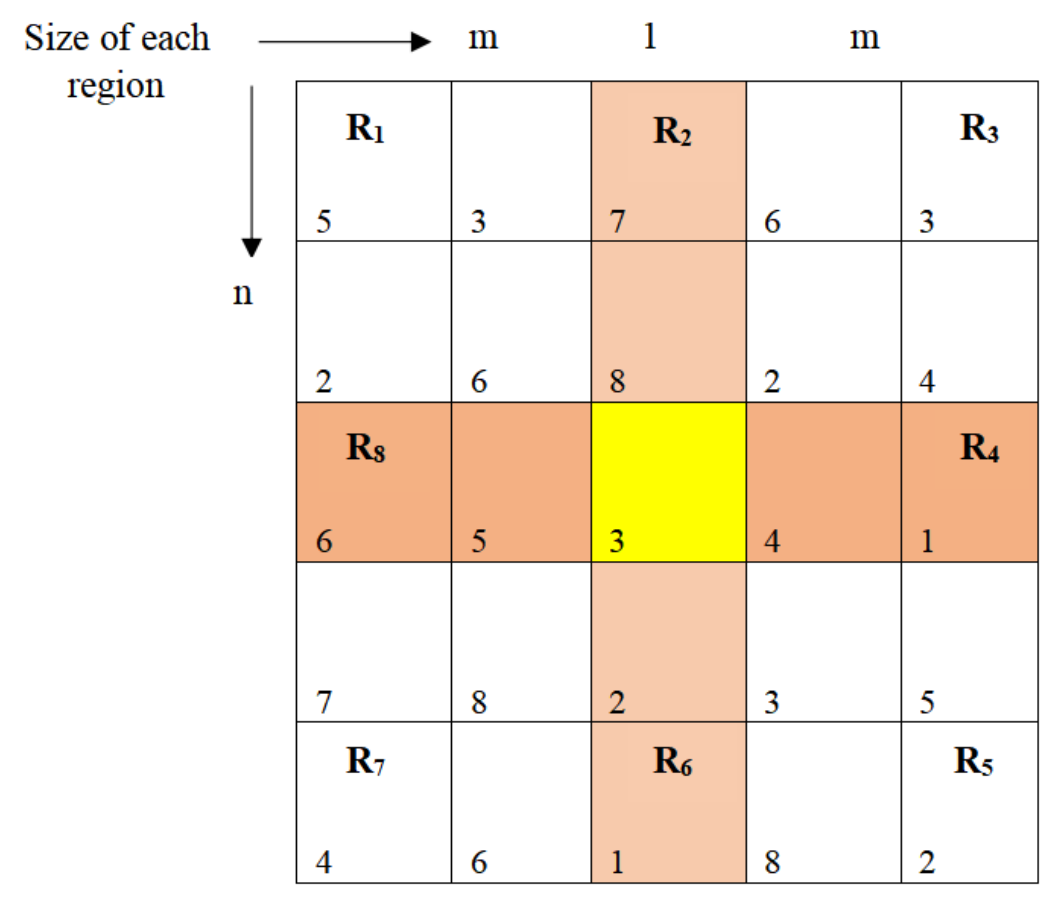

Fig.2. Shows basic 5x5 ARLBP operator

Fig. 2 shows ARLBP operator consisting of eight different sub regions around the central region which are labeled as $R_{i}\{i=1,2,3 \ldots \ldots . .8\}$. The sizes of the regions $R_{1}, R_{3}, R_{5}$ and $R_{7}$ are varying in both horizontal and vertical 
directions. Whereas the sizes of regions $R_{2}$ and $R_{6}$ varies in vertical directions and $R_{4}$ and $R_{8}$ varies in horizontal direction, the central region is of fixed size of $1 \mathrm{x} 1$, whereas the sizes of the sub regions change the size of the operator. In general, ARLBP operator with $(2 \mathrm{~m}+1) \times(2 n+1)$ size consists of four $n \times m$, two $1 \times n$, two mx 1 and central region is of $1 \times 1$ rectangular sized regions where $m$ and $n$ are width and height of the region. Keeping the value $m=1$ and $n=1$, the ARLBP operator is equivalent to basic LBP operator. The ARLBP operator is applied to extract the features by considering $5 \times 5$ window assigning $m=2$ and $n=2$ and average value of the pixel is calculated and rounded off to the nearest integer value.

Fig. 3 shows the averaging of all the sub regions. Example: The average value of the sub region $R_{1}$ is calculated by adding all four pixel values such as $5,3,2$ and 6 and divided by 4 to get the average value of the sub region $R_{1}$. In the sub region $R_{3}$ the average value is 3.75 which are rounded off to the nearest value 4 . In the same manner the average values are calculated for all the sub regions. By averaging the sub region values of the $5 \times 5$ matrix of Fig. 2 is reduced to $3 \times 3$ matrix as shown in Fig.4. The final feature vector is obtained by comparing the neighbor pixel value with central pixel value using relation $\operatorname{ARLBP}\left(x_{c}, y_{c}\right)$.

$$
\operatorname{ARLBP}\left(x_{c}, y_{c}\right)=\sum_{i=1}^{8} S\left(a_{i}-a_{c}\right) 2^{P}
$$

Where $a_{i}$ is the average gray values of the regions $R_{i}\{i=1,2,3 \ldots \ldots . .8\}$ and $a_{c}$ is the central pixel value. The function $S\left(a_{i}-a_{c}\right)$ can be defined as

$$
S\left(a_{i}-a_{c}\right)=\left\{\begin{array}{rr}
1, & \left(a_{i}-a_{c}\right)>0 \quad n \\
0, & \text { Otherwise }
\end{array}\right.
$$

\begin{tabular}{|c|c|} 
Region $R_{1}$ \\
\hline 5 & 3 \\
\hline 2 & 6 \\
\hline
\end{tabular}

$$
R_{1}=\frac{(5+3+6+2)}{4}=4
$$

Region $R_{5}$

\begin{tabular}{|c|c|}
\hline 3 & 5 \\
\hline 8 & 2 \\
\hline
\end{tabular}

$$
R_{5}=\frac{(3+5+8+2)}{4} \cong 5
$$

Region $R_{2}$

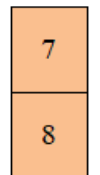

$$
R_{2}=\frac{(7+8)}{2} \cong 8
$$

Region $R_{6}$

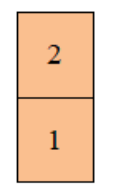

$$
R_{6}=\frac{(2+1)}{2} \cong 2
$$

Region $R_{3}$

\begin{tabular}{|c|c|}
\hline 6 & 3 \\
\hline 2 & 4 \\
\hline
\end{tabular}

$$
R_{3}=\frac{(6+3+2+4)}{4}=\frac{15}{4} \cong 4
$$

Region $R_{7}$

\begin{tabular}{|c|c|}
\hline 7 & 8 \\
\hline 4 & 6 \\
\hline
\end{tabular}

$$
R_{7}=\frac{(7+8+4+6)}{4} \cong 6
$$

Region $R_{4}$

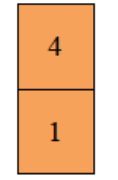

$$
R_{4}=\frac{(4+1)}{2} \cong 3
$$

Region $R_{8}$

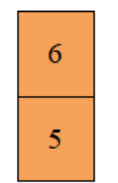

$R_{8}=\frac{(6+5)}{2} \cong 6$

Fig.3. Averaging of the sub regions. 


\begin{tabular}{|c|c|c|}
\hline$R_{1}=4$ & $R_{2}=8$ & $R_{3}=4$ \\
\hline$R_{8}=6$ & 3 & $R_{4}=3$ \\
\hline$R_{7}=6$ & $R_{6}=2$ & $R_{5}=5$ \\
\hline
\end{tabular}

\begin{tabular}{|l|l|l|}
\hline 1 & 1 & 1 \\
\hline 1 & & 1 \\
\hline 1 & 0 & 1 \\
\hline
\end{tabular}

Binary Code : 11111011

Decimal Code : 251

Fig.4. ARLBP Binary code after Thresholding

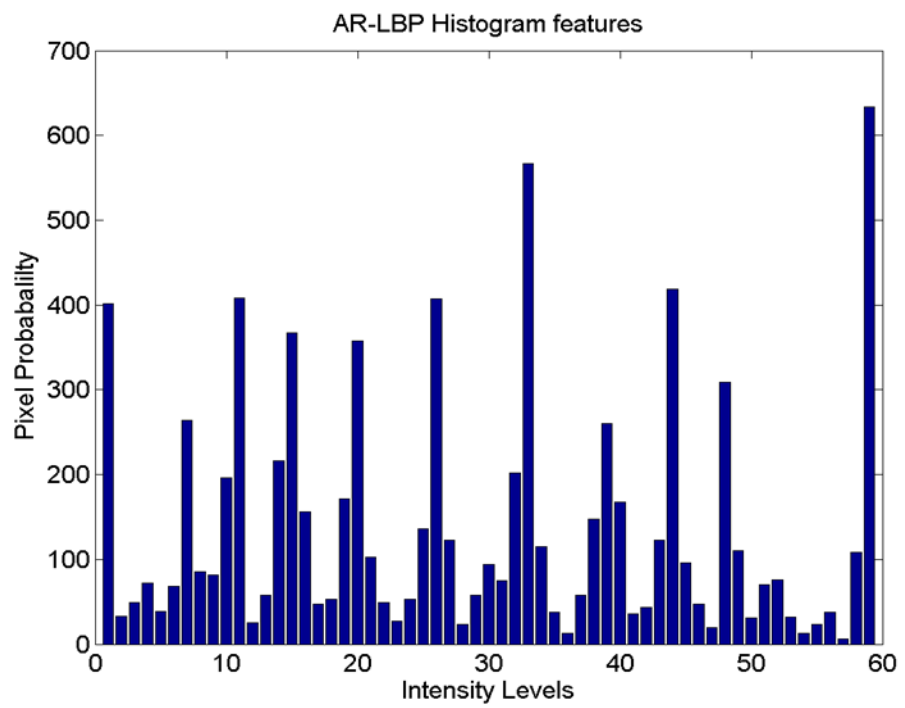

Fig.5. The ARLBP histogram plot obtained for a face image

When central pixel value is 3 and pixel value of the neighbor i.e. $R_{1}$ is 4 which is higher than the central pixel value 3 hence is assigned the binary value 1 , where as in $R_{6}$ the average pixel value is 2 which is less than the central pixel value hence is assigned binary value 0 and so on. The binary code 11111011 is constructed in clockwise direction and converted into equivalent decimal value of 251 and this value is considered as a ARLBP feature value and same procedure is carried out for the entire face image. The histogram descriptor values are calculated on obtained ARLBP features by moving the window on the entire face image as shown in Fig.5. The obtained feature vector is considered as final ARLBP feature vector for matching. Fig.6 and Fig.7 shows the input NIR face images and the corresponding ARLBP output images. Fig.8 and Fig.9 shows the input FERET face images and the corresponding ARLBP output images.

\subsection{FDCT Features}

Fast Discrete Curvelet Transform (FDCT) technique is used to extract the facial features from the preprocessed image in transform domain. The Curvelets via wrapping technique [26] has been used in the proposed work as this technique is simple, faster and less redundant than first generation curvelet transform. The curvelet via wrapping technique is applied on resized images to obtain the coefficients at each wedge. The curvelet coefficients obtained at each wedge is a function of two windows namely radial window $W$ and the angular window $V$. 

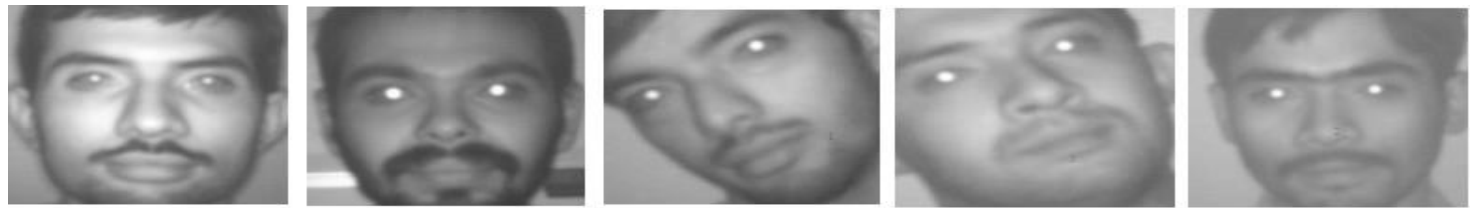

Fig.6. Input face images from NIR Database
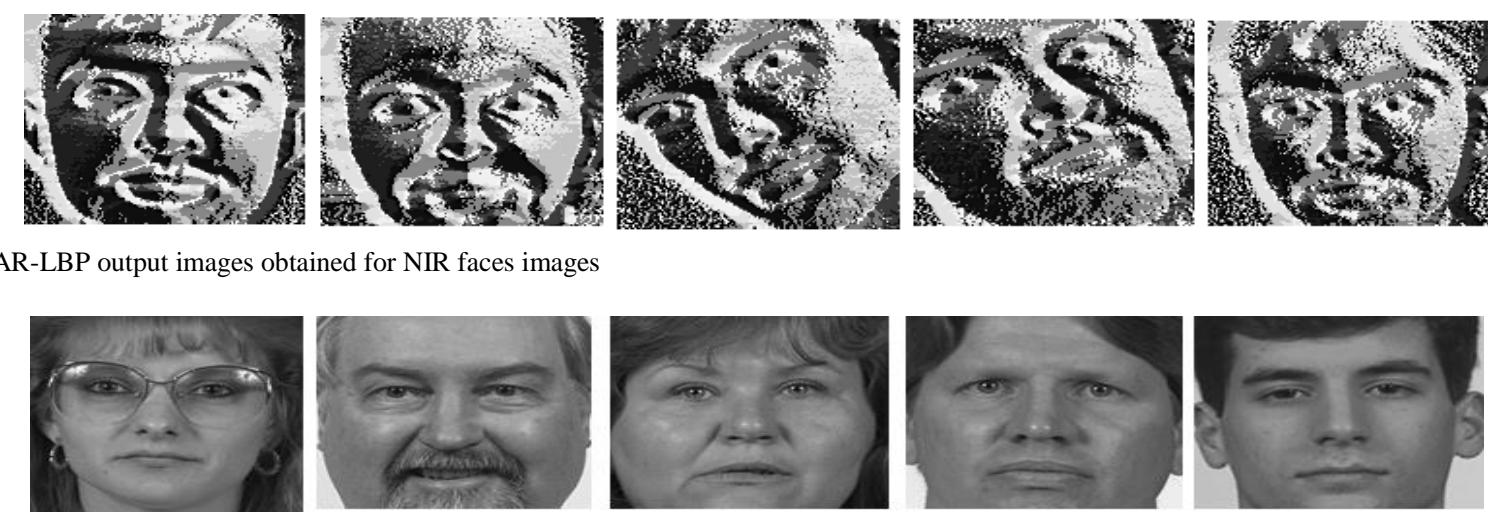

Fig.7. AR-LBP output images obtained for NIR faces images
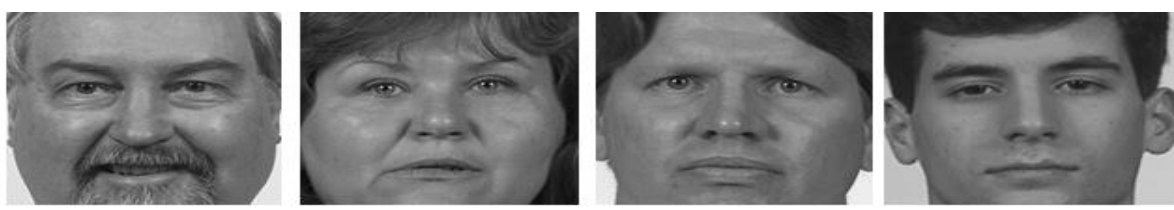

Fig.8. Input face images from FERET Database

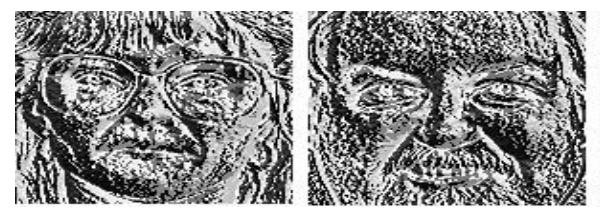

Fig.9. AR-LBP output images obtained for FERET faces images
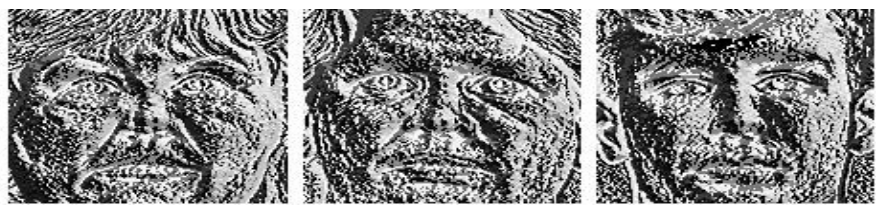

The Cartesian window $\tilde{U}_{j, l}$ isolates frequencies near the wedges $\left(\omega_{1}, \omega_{2}\right)$ is given by

$$
\tilde{U}_{j, l}=W_{j}(\omega) V_{j}(\omega)
$$

Where

$$
\begin{gathered}
W_{j}(\omega)=\sqrt{\varphi_{j+1}^{2}(\omega)-\varphi_{j}^{2}(\omega)}, j \geq 0 \\
\varphi_{j}\left(\omega_{1}, \omega_{2}\right)=\phi\left(2^{-\mathrm{j}} \omega_{1}\right) \phi\left(2^{-\mathrm{j}} \omega_{2}\right), 0 \leq \phi \leq 1 . \\
\mathrm{V}_{\mathrm{j}}(\omega)=\mathrm{V}\left(2^{j / 2} \omega_{1} / \omega_{2}\right)
\end{gathered}
$$

Curvelet via wrapping technique algorithm is developed by considering $f\left(t_{1}, t_{2}\right)$ as an input Cartesian array and $\hat{f}\left(n_{1}, n_{2}\right)$ as a $2 \mathrm{D}$

Discrete Fourier Transform as follows:

1. 2D Fast Fourier Transform (FFT) is applied for input Cartesian array $f\left(t_{1}, t_{2}\right)$ to obtain $\hat{f}\left(n_{1}, n_{2}\right)$.

2. For each angle $l$ and scale $j$, the product $\tilde{U}_{j, l}\left(n_{1}, n_{2}\right) * \hat{f}\left(n_{1}, n_{2}\right)$ is calculated, where $\tilde{U}_{j, l}\left(n_{1}, n_{2}\right)$ is discrete localizing Cartesian window.

3. This product is wrapped around origin to obtain $\hat{f}_{j, l}\left(n_{1}, n_{2}\right)=W\left(\tilde{U}_{j, l} \hat{f}\right)\left(n_{1}, n_{2}\right)$, where $0 \leq n_{1}<L_{1, j}$ and $0 \leq n_{2}<L_{2, j}$ and $L_{1, j} \sim 2^{j}$ and $L_{2, j} \sim 2^{j / 2}$

4. Apply 2D inverse FFT to each $\hat{f}_{j, l}$, to obtain the discrete curvelet coefficients. 
The input face images of size $100 \times 100$ with different intensity maps are considered. The different intensity images are thus obtained by quantizing the image pixels with quantization parameter like 1, 16 and 64 . Then curvelet is applied for each quantized image for different scales till scale $=5$. This results in 5 (scales) $\times 3$ (quantized images) $=$ 15 (feature vectors) and the obtained feature vector is considered as secondary feature vector for matching. Fig.10 shows the curvelet via wrapping technique for L-space $\mathrm{k}$ face image with scale $=5$.

\subsection{Features Fusion}

The features of ARLBP and feature of FDCT are combined by means of concatenation to obtain the final feature set for matching. In the proposed work different classifiers such as Support Vector Machine (SVM), Euclidean Distance (ED), Chi Square, Cosine, City block, Correlation and Spearman are applied. Each of these distance metrics are tested rigorously with ARLBP, FDCT and fusion [ARLBP+FDCT] by concatenation technique and the results are discussed in Results and Discussion Section.

\section{Classification}

Support Vector Machine (SVM): The set of data consists of $N$ samples called training set $\left\{x_{i}, y_{i}\right\}_{i=1}^{N}$, where $x_{i}$ the input samples are, $x_{i} \in \mathcal{R}^{n}$ belongs to one of two classes labeled by $y_{i}= \pm 1$. The SVM classifier outputs based on the decision surface of the form $\operatorname{sign}[f(x ; w)]$,

where $f(x ; w)=w^{T} \phi(x)+b$ with an approximation to the mapping function $y$. The following optimization problem is obtained in order to derive $w$ and $b$.

$$
\begin{gathered}
\min _{w, b, \xi} \phi(w, b, \xi)=\frac{1}{2}\left(w^{T} w\right)+C \sum_{i=1}^{N} \xi \\
\text { Subject to } y_{i}\left[w^{T} \phi(x)+b\right] \geq 1-\xi_{i}, \xi_{i} \geq 0
\end{gathered}
$$

$i=0,1 \ldots \ldots . N$, Where $\mathrm{C}$ represents the trade-off parameter reflecting the relative significance of model's complexity compared to training error and $\xi_{i}$ indicates the training error for $i^{\text {th }}$ sample.

Euclidean Distance (ED): Euclidean Distance (ED), is used since its simple and effective. The Euclidean Distance $d$ for the pair of feature vectors is given by

$$
d=\sqrt{\sum_{i=1}^{N}\left(p_{i}-q\right)^{2}}
$$

$p_{i}$ Feature vector from the Database images

$q$ Feature vector from the Test Image

$N$ Total Images in the Database.

Chi Square distance: The Chi Square distance $d$ for the pair of feature vectors is given by

$$
d=\sum_{i=1}^{N} \frac{\left(p_{i}-q\right)^{2}}{\left(2+p_{i}+q\right)}
$$

$p_{i}$ Feature vector from the Database images

$q$ Feature vector from the Test Image

$N$ Total Images in the Database. 
City block distance: The City block distance $d$ for the pair of feature vectors is given by

$$
d=\sum_{i=1}^{N} a b s\left(p_{i}-q\right)
$$

$p_{i}$ Feature vector from the Database images

$q$ Feature vector from the Test Image

$N$ Total Images in the Database.

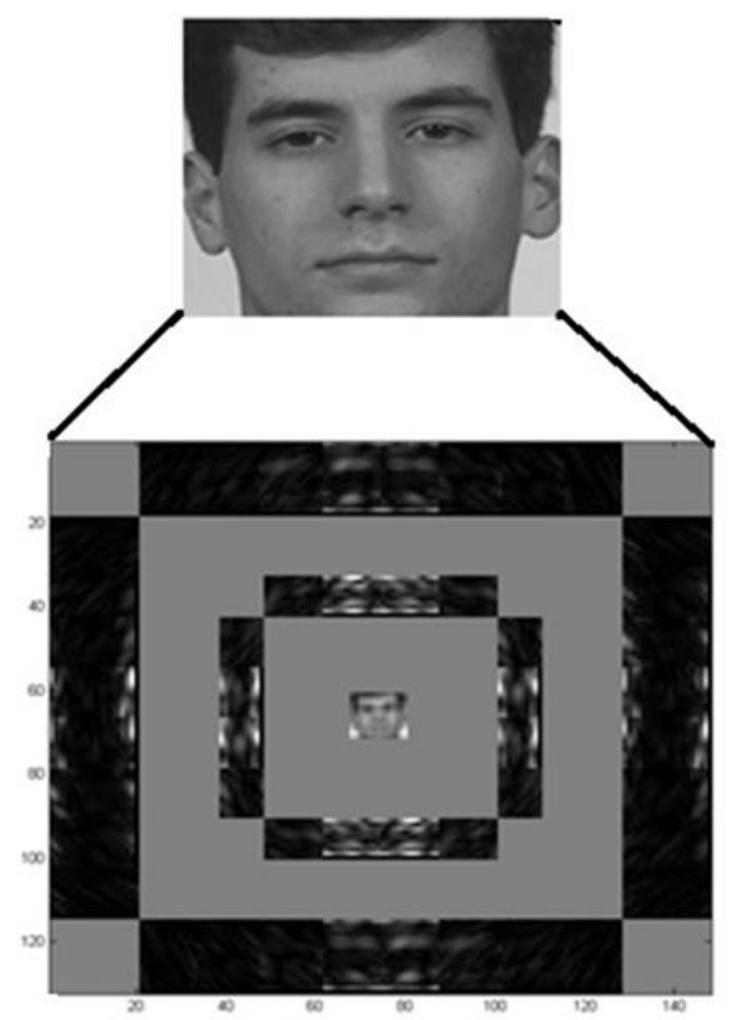

Fig.10. Curvelet via wrapping technique for face image with scale $=5$

Cosine distance: The Cosine distance $d$ for the pair of feature vectors is given by

$$
d=1-\frac{p_{i} \times q^{\prime}}{\sqrt{\left(q \times q^{\prime}\right)\left(p_{i}{ }^{\prime} \times p_{i}^{\prime}\right)}}
$$

$p_{i}$ Feature vector from the Database images

$q$ Feature vector from the Test Image

Correlation distance: The Correlation distance $d$ for the pair of feature vectors is given by

$$
d=1-\frac{\left(p_{i}-\overline{p_{i}}\right) \times(q-\bar{q})^{\prime}}{\sqrt{\left(p_{i}-\overline{p_{i}}\right) \times\left(p_{i}-\overline{p_{i}}\right)^{\prime}} \sqrt{(q-\bar{q}) \times(q-\bar{q})^{\prime}}}
$$

$p_{i}$ Feature vector from the Database images

$q$ Feature vector from the Test Image 


$$
\overline{p_{i}}=\frac{1}{N} \sum_{j=1}^{N} p_{i j} \quad \bar{q}=\frac{1}{N} \sum_{j=1}^{N} q_{j}
$$

$N$ Total Images in the Database.

Spearman distance: The Spearman distance $d$ for the pair of feature vectors is given by

$$
d=1-\frac{\left(r_{s}-\overline{r_{s}}\right) \times(r-\bar{r})^{\prime}}{\sqrt{\left(r_{s}-\overline{r_{s}}\right) \times\left(r_{s}-\overline{r_{s}}\right)^{\prime}} \sqrt{(r-\bar{r}) \times(r-\bar{r})^{\prime}}}
$$

$r_{s j}$ is the rank of $p_{i j}$ taken over $p_{1 j}, p_{2 j}, \ldots \ldots \ldots p_{m j} r_{s}$ and $r$ are the coordinate-wise rank vectors of $p_{i}, q$.

$$
\overline{r_{s}}=\frac{1}{N} \sum_{j=1}^{N} r_{s j} \quad \bar{r}=\frac{1}{N} \sum_{j=1}^{N} r_{j}
$$

$\underline{\text { Algorithm }}$

Input: Image of Face.

Output: Recognized Face of Person.

Step 1: Face image has been selected from the database for reading.

Step 2: Face region is detected from the input image using Viola Jones Algorithm.

Step 3: The detected face image is cropped and resized to $100 \mathrm{X} 100$ dimensions.

Step 4: Asymmetric Region Local Binary Pattern (ARLBP) is applied over the face image to extract spatial domain features.

Step 5: Curvelet via wrapping is applied on detected face to extract transform domain feature coefficients.

Step 6: The features from ARLBP and FDCT are combined by concatenation to form final feature vector.

Step 7: Repeat the above procedure for test image from step 1 to 6

Step 8: Test image features were compared with Database image features using different classifiers for ARLBP, FDCT and fusion of ARLBP and FDCT for matching.

\section{Results and Discussions}

The proposed face recognition system has been evaluated on different databases such as L-Spacek, NIR, JAFFE and FERET. The performance is evaluated by computing Recognition Rate for different threshold values.

Recognition Rate: It is measure in biometric system. Recognition Rate increases if a system correctly accepts an access to authorized person and it can be given as

$$
\text { Recognition Rate }=\frac{\text { Number of genuine samples }}{\text { Total number of Images in Database }}
$$

In Table 1 recognition rate is tabulated for all the databases. The percentage recognition rate is measured individually using FDCT and ARLBP feature extraction techniques and also by fusing the FDCT features and ARLBP features by concatenation to improve the performance of the proposed system on the preprocessed databases. It is notable that the percentage recognition rate is better in the fusion technique compared to individual ARLBP and FDCT feature extraction technique for all the distance measure as well as SVM classifier. The hybrid technique better recognition rate $95.48 \%$ for FERRET, $92.18 \%$ for $\mathrm{L}$ space $\mathrm{k}, 76.55 \%$ for JAFFE and $81.44 \%$ for NIR respectively using SVM classifier.

Table 2 shows the percentage recognition rate of individual ARLBP and FDCT technique and hybrid technique [FDCT +ARLBP] algorithm compared with the existing algorithm [27] for FERET database with image size of $130 \times 150$ with different descriptors and distance measures. Further, the proposed work is also compared with the existing algorithms for the NIR database [30] and FERET database [28, 29, 31, 32, 33] as shown in Table 3. It is found that the proposed system has better recognition rate. 
Table 1. Recognition rates (\%) using various feature extraction techniques vs. different distance measures/classifier for face recognition on different database

\begin{tabular}{|c|c|c|c|c|c|c|c|}
\hline Method & SVM & ED & $\begin{array}{c}\text { Chi } \\
\text { Square }\end{array}$ & $\begin{array}{c}\text { City } \\
\text { Block }\end{array}$ & Cosine & $\begin{array}{l}\text { Corre } \\
\text { lation }\end{array}$ & $\begin{array}{c}\text { Spear } \\
\text { man }\end{array}$ \\
\hline \multicolumn{8}{|c|}{ FERET Database } \\
\hline $\begin{array}{l}\text { ARLBP } \\
\text { FDCT } \\
\text { FDCT+ } \\
\text { ARLBP }\end{array}$ & $\begin{array}{l}89.14 \\
90.14 \\
95.48\end{array}$ & $\begin{array}{l}85.66 \\
86.66 \\
93.33\end{array}$ & $\begin{array}{l}85.78 \\
86.88 \\
93.33\end{array}$ & $\begin{array}{l}86.14 \\
85.48 \\
93.33\end{array}$ & $\begin{array}{l}85.66 \\
86.14 \\
93.48\end{array}$ & $\begin{array}{l}84.14 \\
86.66 \\
92.18\end{array}$ & $\begin{array}{l}86.66 \\
86.10 \\
93.88\end{array}$ \\
\hline \multicolumn{8}{|c|}{ L-spacek Database } \\
\hline $\begin{array}{l}\text { ARLBP } \\
\text { FDCT } \\
\text { FDCT+ } \\
\text { ARLBP }\end{array}$ & $\begin{array}{l}88.89 \\
90.16 \\
92.18\end{array}$ & $\begin{array}{l}87.44 \\
89.26 \\
90.33\end{array}$ & $\begin{array}{l}83.87 \\
86.10 \\
87.77\end{array}$ & $\begin{array}{l}85.66 \\
88.88 \\
89.03\end{array}$ & $\begin{array}{l}86.33 \\
87.89 \\
88.96\end{array}$ & $\begin{array}{l}84.33 \\
88.17 \\
89.00\end{array}$ & $\begin{array}{l}82.88 \\
86.33 \\
88.67\end{array}$ \\
\hline \multicolumn{8}{|c|}{ JAFFE Database } \\
\hline $\begin{array}{c}\text { ARLBP } \\
\text { FDCT } \\
\text { FDCT+ } \\
\text { ARLBP }\end{array}$ & $\begin{array}{l}68.66 \\
70.14 \\
76.55\end{array}$ & $\begin{array}{l}66.88 \\
69.44 \\
71.22\end{array}$ & $\begin{array}{l}65.33 \\
67.33 \\
68.77\end{array}$ & $\begin{array}{l}64.55 \\
68.77 \\
70.09\end{array}$ & $\begin{array}{l}66.88 \\
65.98 \\
67.96\end{array}$ & $\begin{array}{l}66.00 \\
68.66 \\
69.55\end{array}$ & $\begin{array}{l}62.95 \\
64.55 \\
66.87\end{array}$ \\
\hline \multicolumn{8}{|c|}{ NIR Database } \\
\hline $\begin{array}{l}\text { ARLBP } \\
\text { FDCT } \\
\text { FDCT+ } \\
\text { ARLBP }\end{array}$ & $\begin{array}{l}70.66 \\
56.67 \\
81.44\end{array}$ & $\begin{array}{l}69.88 \\
52.44 \\
80.11\end{array}$ & $\begin{array}{l}64.67 \\
50.88 \\
78.33\end{array}$ & $\begin{array}{l}62.11 \\
49.99 \\
79.82\end{array}$ & $\begin{array}{l}61.88 \\
50.77 \\
78.09\end{array}$ & $\begin{array}{l}67.88 \\
51.83 \\
79.34\end{array}$ & $\begin{array}{l}60.09 \\
48.77 \\
77.55\end{array}$ \\
\hline
\end{tabular}

Table 2. Comparison of Recognition rates (\%) using various feature extraction techniques vs. different distance Measures /classifier for face recognition on FERET database of image size $130 \times 150$.

\begin{tabular}{|c|c|c|c|c|c|c|c|}
\hline Method & SVM & ED & $\begin{array}{c}\text { Chi } \\
\text { Square }\end{array}$ & $\begin{array}{c}\text { City } \\
\text { Block }\end{array}$ & Cosine & Correlation & $\begin{array}{c}\text { Spear } \\
\text { man }\end{array}$ \\
\hline FERET Database \\
\hline LBP [27] & 42.3 & 60.9 & 77.1 & 73.3 & 60.9 & 60.7 & 66.1 \\
LPQ [27] & 77.3 & 77.2 & 79.4 & 78.3 & 77.3 & 77.1 & 66.8 \\
HOG [27] & 87.2 & 88.1 & 85.8 & 88.3 & 83.4 & 84.7 & 86.7 \\
\hline ARLBP & 89.1 & 85.7 & 85.8 & 86.1 & 85.7 & 84.1 & 86.7 \\
FDCT & 90.1 & 86.7 & 86.9 & 85.5 & 86.1 & 86.7 & 86.1 \\
FDCT+ ARLBP & 95.5 & 93.3 & 93.3 & 93.3 & 93.5 & 92.2 & 93.9 \\
[Proposed] &
\end{tabular}

Table 3. Comparison of Recognition rates (\%) with previous studies for different database images

\begin{tabular}{|c|c|c|}
\hline References & Database & Accuracy in $\%$ \\
\hline$[28]$ & FERET & 91.0 \\
\hline$[29]$ & FERET & 91.2 \\
\hline$[30]$ & NIR & 60.0 \\
\hline$[31]$ & FERET & 78.0 \\
\hline$[32]$ & FERET & 76.8 \\
\hline$[33]$ & FERET & 95.0 \\
\hline$[$ Proposed] & FERET & 95.48 \\
\hline$[$ Proposed] & NIR & 81.44 \\
\hline
\end{tabular}

\section{Conclusion}

The proposed face recognition system performs both in spatial as well as frequency domain feature extraction techniques. The spatial feature extraction is achieved using Asymmetric Region Local binary pattern (ARLBP) and frequency domain feature extraction using Fast Discrete Curvelet Transform (FDCT). The preprocessing is carried out on the face region in every image by cropping and resized for a fixed dimension of size 100x100. The experiment is also conducted individually for ARLBP and Curvelet alone. To improve the recognition rate the features of ARLBP and FDCT are combined by concatenation. For matching different distance metrics and SVM classifier are used to measure the recognition rate for different databases such as FERET, L-Space k, JAFFE and NIR. The results shows that the performance of the hybrid technique is superior when compare to the individual technique alone. The Recognition rate is considered as an assessment parameter to evaluate the performance of the algorithm. It is observed that the recognition rate is better for combining both ARLBP and FDCT techniques than that of individual techniques alone. 


\section{Acknowledgment}

The research was supported by Visvesvaraya Technological University, Jnana Sangama, Belagavi - 590018, Karnataka, India.

\section{References}

[1] Marcos Faundez-Zanuy, "Biometric Security Technology," Encyclopedia of Artificial Intelligence, Vol. 1, pp. 262-264, Jan. 2008.

[2] Aftab Ahmed, Jiandong Guo, Fayaz Ali, Farha Deeba and Awais Ahmed, "LBPH Based Improved Face Recognition at Low Resolution," International Conference of Artificial Intelligence and Big Data, pp. 144-147, May. 2018. DOI: 10.1109/ICAIBD.2018.8396183.

[3] Katarina Knezevic, Emilija Mandic, Ranko Petrovic, and Branka Stojanovic, "Blur and Motion Blur Influence on Face Recognition Performance," symposium on Neural Networks and Applications, pp. 1-5, Nov 2018. DOI: 10.1109/NEUREL.2018.8587028.

[4] Teddy Mantoro, Media A. Ayu and Suhendi, "Multi-Faces Recognition Process Using Haar Cascades and Eigen face Methods," International Conference on Multimedia Computing and Systems, pp. 1-5, Mar. 2018. DOI: 10.1109/ICMCS.2018.8525935.

[5] M. Sushama and E. Rajinikanth, "Face Recognition using DRLBP and SIFT Feature Extraction," International Conference on Communication and Signal Processing, pp. 994-999, Apr. 2018. DOI: 10.1109/ICCSP.2018.8524427.

[6] Gaili Yue and Lei Lu, "Face recognition based on histogram equalization and convolution neural network," International Conference on Intelligent Human-Machine Systems and Cybernetics, pp. 336-339, Aug. 2018. DOI: 10.1109/IHMSC.2018.00084.

[7] XueMei Zhao and ChengBing Wei, “A Real-time Face Recognition System Based on the Improved LBPH Algorithm,” IEEE International Conference on Signal and Image Processing, pp. 72-76, Aug. 2017. DOI: 10.1109/SIPROCESS.2017.8124508.

[8] Pattarakamon Rangsee, K B Raja and Venugopal K R, “ Modified Local Ternary Pattern Based Face Recognition using SVM,” Image Processing, Computer Science and Information technology , pp. 343-350, Oct. 2018.DOI: 10.1109/ICIIBMS.2018.8549952.

[9] Ranjana Sikarwar, Arun Agrawal and Rajendra Singh Kushwah, "An Edge Based Efficient Method of Face Detection and Feature Extraction," International Conference on Communication Systems and Network Technologies, pp. 1147-1151, Apr. 2015. DOI: 10.1109/CSNT.2015.167.

[10] Kennedy Chengeta and Serestina Viriri, “A Survey on Facial Recognition based on Local Directional and Local Binary Patterns," Conference on Information Communications Technology and Society, pp. 1-6, Mar. 2018.DOI: 10.1109/ICTAS.2018.8368757.

[11] Liu Tianyu, Li Fei and Wang Rui, "Human Face Gender Identification System Based on MB-LBP," Chinese Control and Decision Conference, pp. 1721-1725, Jun. 2018.DOI: 10.1109/CCDC.2018.8407405.

[12] Priya Gupta, Nidhi Saxena, Meetika Sharma, Jagriti Tripathi,"Deep Neural Network for Human Face Recognition", International Journal of Engineering and Manufacturing, Vol.8, No.1, pp.63-71, 2018.DOI: 10.5815/ijem.2018.01.06.

[13] Ashirbani Saha and Q.M.Jonathan Wu, "Facial expression recognition using curvelet based Local Binary Patterns," IEEE International Conference on Acoustics, Speech and Signal Processing, pp. 2470-2473, Mar. 2010.DOI: 10.1109/ICASSP.2010.5494892.

[14] Huda Mady and Shahid M .S. Hilles, "Face recognition and detection using Random forest and combination of LBP and HOG features," International Conference on Smart Computing and Electronic Enterprise, pp. 1-7, Jul. 2018.DOI: 10.1109/ICSCEE.2018.8538377.

[15] Dr. Eyad I. Abbas, Mohammed E. Safi- MIEEE and Dr. Khalida S. Rijab, "Face Recognition Rate Using Different Classifier Methods Based on PCA," International Conference on Current Research in Computer Science and Information Technology, pp. 37-40, Apr. 2017.DOI: 10.1109/CRCSIT.2017.7965559.

[16] Marko Arsenovic, Srdjan Sladojevic, Andras Anderla and Darko Stefanovic, "Face Time - Deep Learning Based Face Recognition Attendance System," IEEE International Symposium on Intelligent Systems and Informatics, pp. 53-58, Sep. 2017.DOI: 10.1109/SISY.2017.8080587.

[17] Sun Feifei, He Min, Nie Rencan and Wu Zhangyong, "Noisy faces recognition based on PCNN and PCA," IEEE International Conference on Electronic Measurement \& Instruments, pp. 300-304, Oct. 2017.DOI: 10.1109/ICEMI.2017.8265797.

[18] Sandip Joardar, Dwaipayan Sen, Diparnab Sen, Arnab Sanyal and Amitava Chatterjee, "Pose Invariant Thermal Face Recognition using Patch-wise Self-similarity Features," International Conference on Research in Computational Intelligence and Communication Networks, pp. 203-207, Nov. 2017.DOI: 10.1109/ICRCICN.2017.8234507.

[19] Narpat A. Singh, Manoj B. Kumar, Manju C. Bala, "Face Recognition System based on SURF and LDA Technique", International Journal of Intelligent Systems and Applications, Vol.8, No.2, pp.13-19, 2016. DOI: 10.5815/ijisa.2016.02.02.

[20] Chang Liu, Jingbing Li and Yucong Duan, "A Face Image Recognition Algorithm based on DFT Encryption Domain," International Conference on Electronics Instrumentation \& Information Systems, pp. 1-6, Jun. 2017.DOI: 10.1109/EIIS.2017.8298669.

[21] Turgut Ozseven and Muharrem Dugenci, "Face Recognition by Distance and Slope between Facial Landmarks," International Artificial Intelligence and Data Processing Symposium, pp. 1-4, Sep. 2017.DOI: 10.1109/IDAP.2017.8090258.

[22] Rabul Saikia and Dr. Aditya Bihar Kandali, "DWT-ELBP based Model for Face Recognition," International Conference on Energy, Communication, Data Analytics and Soft Computing, pp. 1348-1352, Aug. 2017.DOI: 10.1109/ICECDS.2017.8389663.

[23] Ravi J, Saleem S Tevaramani and K B Raja, "Face Recognition using DT-CWT and LBP Features," International Conference on Computing, Communication and Applications, pp. 1-6, Feb. 2012.DOI: 10.1109/ICCCA.2012.6179224.

[24] Paul Viola and Michael J. Jones, “ Robust Real-Time Face Detection,” International Journal of Computer Vision, Vol. 57, No. 2, 
pp. 137-154, 2004. DOI: https://doi.org/10.1023/B:VISI.0000013087.49260.fb.

[25] C. L. Shrinivasa Naika, Pradip K. Das , Shivashankar B. Nair, "Asymmetric region Local Binary Pattern operator for persondependent facial expression recognition", International Conference on Computing, Communication and Applications, pp. $1-5$, Feb. 2012.DOI: 10.1109/ICCCA.2012.6179199.

[26] Emmanuel Candes, Laurent Demanet,David Donoho and Lexing Ying, "Fast Discrete Curvelet Transforms," Multiscale Modeling \& Simulation, Vol. 5, pp. 861-899, Sep. 2006. DOI: 10.1137/05064182X.

[27] Bappaditya Mandal, Zhikai Wang, Liyuan Li, Ashraf A. Kassim, "Performance evaluation of local descriptors and distance measures on benchmarks and first-person-view videos for face identification," Neurocomputing, Vol.184, Issue C, pp. 107-116, Apr. 2016. DOI: 10.1016/j.neucom.2015.07.121.

[28] Rahul Varma, Sandesh Gupta and Phalguni Gupta, "Face Recognition system invariant to expressions", International Conference on Intelligent Computing, Springer, pp.299-307, 2014. DOI: https://doi.org/10.1007/978-3-319-09333-8_33.

[29] Shih-Ming Huang and Jar-Ferr Yang, Fellow, "Linear Discriminant Regression Classification for Face Recognition",IEEE signal processing letters, Vol. 20, No. 1, Jan. 2013. DOI: 10.1109/LSP.2012.2230257.

[30] Faten Omri, Sebti Foufou and Mongi Abidi, "NIR and Visible Image Fusion for Improving Face Recognition at Long Distance", International Conference on Image and Signal Processing,pp 549-557, 2014. DOI: https://doi.org/10.1007/978-3-319-079981 63.

[31] Yongbin Qin, Lilei Sun and Young $\mathrm{Xu}$, "Exploring of alternative representations of facial images for face recognition,"International Journal of Machine Learning and Cybernetics, pp. 1-7, Apr. $2020 . \quad$ DOI: https://doi.org/10.1007/s13042-020-01116-4.

[32] Surabhi Gupta, Kutub Thakur and Munish Kumar, "2D-human face recognition using SIFT and SURF descriptors of face's feature regions," The Visual Computer, pp. 1-10, Feb. 2020. DOI: https://doi.org/10.1007/s00371-020-01814-8.

[33] Turker Tuncer, Sengul Dogan, Erhan Akbal, " Discrete Complex Fuzzy Transform based Face Image Recognition Method", International Journal of Image, Graphics and Signal Processing, Vol.11, No.4, pp. 1-7, 2019.DOI:10.5815/ijigsp.2019.04.01.

\section{Authors' Profiles}

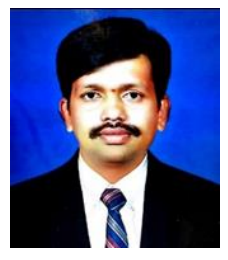

Raveendra $\mathbf{K}$ is a Assistant Professor in the Department of Electronics and Communication Engineering, Government Engineering College, K R Pet. He obtained his BE Degree in Electronics and Communication Engineering from Kuvempu University, Shimoga His specialization in Master degree was Digital Communication Engineering from Visvesvaraya Technological University, Belagavi.

$\mathrm{He}$ is pursuing PhD in Electronics and Communication Department, Global Academy of Technology, Bengaluru. His area of interest is in the field of Digital Image Processing, Communication and Digital Signal Processing and Biometrics. He is life member of ISTE, New Delhi.

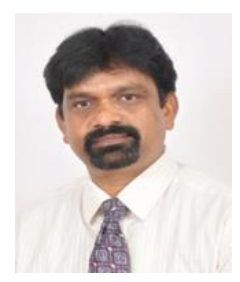

Dr. Ravi J is a Professor in the Department of Electronics and Communication Engineering, Global Academy of Technology, Bangalore. He obtained his BE Degree in Instrumentation Technology from Bangalore University, Bangalore. His specialization in Master degree was Digital Electronics from Visvesvaraya Technological University, Belagavi.

He was awarded PhD in Electronics and Communication Engineering from JNTU Anantapur. He has over 30 research publications in refereed International Journals and Conference Proceedings. His research interests include Image Processing, Biometrics, VLSI, Signal Processing and computer networks. He is life member of IETE, ISTE, and ISAMP.

How to cite this paper: Raveendra K, Ravi J, "Performance Evaluation of Face Recognition system by Concatenation of Spatial and Transformation Domain Features", International Journal of Computer Network and Information Security(IJCNIS), Vol.13, No.1, pp.47-60, 2021. DOI: 10.5815/ijcnis.2021.01.05 